\title{
Component Missing
}

National Cancer Institute

\section{Source}

National Cancer Institute. Component Missing. NCI Thesaurus. Code C63021.

A device component(s) found to be missing when delivered to the user facility. 